\title{
Distributed Data Storage Technique for Big Data using Hadoop
}

\author{
M.M. Kodabagi, Savita Rathod and Vilas Naik
}

\begin{abstract}
Nowadays the data size and domains of data is increasing because of the use by both public and private sectors. The size of the data is increasing every year by double the rate. There are mainly three types of data structured, unstructured and semi-structured data. The existing systems were unable to store the structured, unstructured and semi-structured data in a single database, Big data is used to store such data. As day by need data size in increasing we need storage management in Big data.
\end{abstract}

The objective of the proposed work is to design HDFS based distributed data storage for Big data, contains different types of Multimedia data such as text, audio, images, Log files and web based data.

In proposed work there are three nodes one is master and two are slaves. Mainly data is stored in master and in both slaves data is distributed to avoid loss of data. Users can access the data from nearest slave Incase one of the slave fails data can be accessed from other slaves. If both slaves fails data can be retrieved from master.

Keywords--- Big data, Hadoop , HDFS, MapReduce.

\section{INTRODUCTION}

$\mathrm{E}$ VERY day nearly 2.5 quintillion bytes of information is made and information creation will be expanding. There are many sources from which data is collected such as information from social networks, every day transaction records, cell and GPS information etc.

Big data refers to a combination of data with varying size and increment in the data makes it non manageable to store and manage. Big data is combination of structured, unstructured and semi-structured data. Whose size ranges from terabytes to petabytes. Big data uses Hadoop platform for data storing, processing and managing. It will manage the large quantity of data.

Around 2.5 quintillion bytes of data is created by users in a single day. Every year information creation is increasing in large quantity. The purpose behind information creation is there are many sources of information such as sensors, social networking, cell and GPS information. This part of

M.M. Kodabagi, Professor, Computer Science \& Engineering, Reva University, Bangalore, India. E-mail:mmkodabagi@gmail.com

Savita Rathod, Student, Computer Science \& Engineering , BEC's Engineering College, Bagalkot, India.E-mail:srsavita3@gmail.com

Vilas Naik, Associate Professor, Computer Science \& Engineering, BEC's Engineering College, Bagalkot, India. Email:vilasnaik_h@rediffmail.com

DOI:10.9756/BIJSESC.8240 information is called Big Data. Big Data is a technology to store, capture, distribute manage and analyze data large size data of different types and at high speed.

Data comes from various sources in different formats and arrive at various rates. To process such data in an efficient way parallelism is used.

Hadoop is the commonly used software by Big Data. Hadoop is software that uses the technique of distributing of data across clusters of slave machines. It can range from a single master machine to thousands of machines with fault tolerance. Data may be in any form such as unstructured, semi-structured, structured, and heterogeneous. Hadoop and HDFS are used for storage and management of Big Data. Analysis of Big data accepts large distributed file system and data should be flexible, scalable and fault tolerant. Analysis of the data is performed by Map Reduce. Map Reduce uses different techniques for mapping, reducing, searching, shuffling and sorting from DBMS.

A. Advantages of using Hadoop

1. Ability to store and process huge amounts of any kind of data, quickly. It will store data from many platforms such as social media (facebook, gmail etc), internet.

2. Computing power. Hadoop processes data fast because of distributed storage of data. Processing power will increase.

3. Fault tolerance. Data is distributed to number of nodes and if one of the node becomes dead then retrieve data from the other node using replication factor.

4. Flexibility. There is no need to preprocess the data for storing. It scales linearly by adding extra nodes. Not much maintance needed.It is less prone to failure of data and simple to handle.

B. Characteristics of Big data

1. Volume:It indicates the amount of data generated and stored. The capacity of Big data ranges from terabytes to perabytes.

2. Velocity:It indicates the rate at which data is generated, stored and processed.

3. Variety:Big data collects a data from various resources such as social media, sensors, web, user interactions and review sites. Data can be structured, unstructured or semi-structured.

4. Complexity:Data management will become a complex process when large volume of data comes from many sources.

5. Variability:Inconsistency of the data set can hamper processes to handle and manage it. 
6. Veracity:The quality of the data being captured can vary greatly. Accuracy of analysis depends on this.

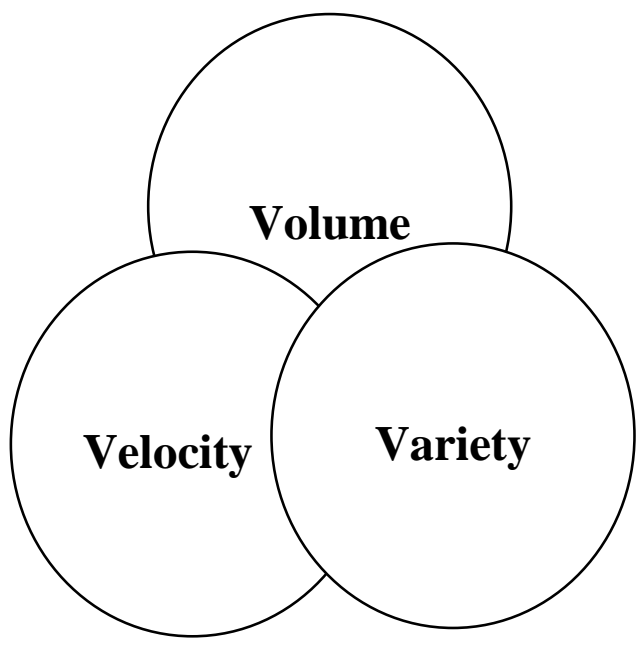

Figure 1: Charecteristics of Big Data

\section{Classification of Big Data}

There are three types of data such as structured, Unstructured and semi-structured data. Data is categorized based on the type of data generated.

\section{Applications}

Nowadays Big data has become a major field in technology. It is being used in many fields because of the feature of having ability to store unstructured data and size of the data is growing continuously. Here there are few Big Data application.

1. Fraud detection: It can be used in real world for analysis of transaction and detecting the behaviour of users. It will help in detection of fraud.

2. IT $\log$ analytics: With Big data we can identify pattern and prevents problems from occurring. It is mainly useful in diagnosing the data.

3. Call center analytics: Big data is used in call centers, so that they can provide the data to the customers early. On call it will identify customer problems and commonly occurring problems.

4. Retail: Big data is being used by many online websites like amazon, tweeter, to reduce the risk of management of data.

5. Entertainment: Big data is used by many entertainment applications such as video game advertisings and predicting performance during game play.

6. Government: Big data is being used by government in analysis of governmental processes.

7. Healthcare: Using Big data we can improve by maintaining a detailed information about patient such as prescriptions, reports, patient registries and prescriptive analysis of record.

8. Media: Nowadays the industry has moved away from using media environments such as newspapers, magazines and televisions.
9. Sports: Big data is used to improve, understand his competitors and can also predict winners by using Big data analytics. It can also be used to predict players future performance.

In this chapter studied the introduction, advantages, characteristic, classification and applications of Big data.

\section{LITERATURE SURVEY}

Here studied few of the prominent research papers to understand the storage of data in Big data.

Jaseena K.U and Julie M.Davis(2014), describes how data is growing every minute. In this papers highlighted the issues and challenges faced for data storage such as privacy, complexity and timeliness.

M.H.Padgavankar, Dr.S.R.Gupta(2014), elaborated about the problems faced by Big data in the industry, Science, Education sector. Few of the challenges listed here are Data representation, Redundancy reduction and data compression, Data life cycle management, Analytical mechanism, Privacy and security.

Ranjana Bahri(2015), describes the fundamental concepts of Big Data. Discussed mainly about the challenges faced by Big data such as capturing, storing, searching, transferring and analysis of data.

Xuebin Chen, Shi Wang, Yanyan Dong, and Xu Wang(2015), presented a "Big data storage architecture in cloud computing". Implement a design which will recover the data from loss and balance the load between slave nodes. Overall provided how storage management can be implemented.

Anusha B. Dhakite , Prof. Sameer Y. Thakur (2015), describes why Hadoop became a powerful tool for analysis and transformation of data. It can store unstructured data and it can distribute across ' $\mathrm{n}$ ' number of machines. Hadoop has inbuilt feature of replication and it will replicate on itself. Presented a architecture of Hadoop layer and explained how task is divided between layers and how data is distribute among 'n' machines.

Yan LIU, Tianjian ZHENG, Mingjiang LI, Jinpeng YUAN(2015), explained how hierarchical storage system is designed.

Praveen Kumar, Dr Vijay Singh Rathore (june 2014), explained the need to develop a efficient system and considering the issues and challenges associated with Big data.

Radhakrishnan R, Karthik S (sep 2014), proposed "heterogeneous file system based storage and retrieval". There will be a single name node server with data distributed and replicated among the data nodes. Presented a system for showing how this system will work.

Yang Li, Li Guo and Yike Guo, described the "design and implementation of CACSS". CACSS architecture is efficient, durable, reliable and scalable. CACSS system includes different components for storage management. 
Rogério Rossi \& Kechi Hirama (2015), presented a Big data management. There are many actors involved in the system. It will provide a pipeline of Big data.

Rabi Prasad Padhy (Feb 2015), discussed how Hadoop and MapReduce can be used for data processing. Data can be produced by many businesses in large quantities. This increase in volume of data has led to the processing and analyzing of information.

Above describes the literature survey of different authors and identified few of the issues and challenges faced by Big data. Processing of data using the existing tools was difficult. Standard tools are not designed for processing and analysing Big data. So organizations encountered early challenges in creating, managing and manipulating large datasets.

Heterogeneity: Humans can tolerate heterogeneity in information but systems need homogeneous data. Data must be structured carefully. There is no fixed format. Data can be in any of the forms .

Incompleteness: The main problem of analysis is the presence of heterogeneous data. More than $80 \%$ of data generated is unstructured. And it is dynamic. There is no fixed format for it. Incomplete data creates uncertainties and it needs to be managed. Incomplete data generally have missing values for samples. Missing values leads malfunctioning of sensor nodes.

1. Scale: As size of the data increases it will become difficult to manage and existing tools were unable to manage data.

2. Timeliness: As the size of data increases it takes more time to process and analyse. As data size increases, time to analyse also increases

3. Personal Privacy: It is an important concern. Privacy of data is a technical problem. There should not to be fear of using data by others.

4. Data confidentiality: It can provide access to other analysis tools only when proper preventive measures are taken to avoid risk of confidentiality when we are sharing confidential data.

5. Storage Issues: There is the increase in quantity of data we are generating daily and it may be due to social media as it is being used by everyone today. The current systems can store 4 terabytes of data and storing and transporting data will overwhelm the network by taking longer time.

6. Management Issues: It the main issue comes into existence after storing the data. We need to manage the stored data and in this case manage data access, utilization and updating. We need to manage the outliers from validating the data. New approaches for validation are needed.

7. Security: In some domains such as facebook such case privacy of the data and security are the commonly raised questions.

In this identified the issues and challenges faced by Big data. In storage of data there are issues like privacy, security, Data loss. In this architecture loss of data is overcome by adding replication factor.

\section{PROPOSED MODEL}

In earlier approach a computer will store and process big data. They used Database storage system for storing data. Due to the huge amount of different types of data it leads to performance database bottleneck. To overcome that Big data system came into existence for storing Heterogeneous data.

In centralized environment there is only one master machine that holds all data means centralized storage. If centralized storage fails then whole system fails. But in Hadoop environment the storage done in distributed way. There is only metadata that hold whole information about the data which is stored on different location. Here metadata called NameNode.

The name node backup will be taken Secondary Namenode after some interval of time it will store to SSN. Here once we want to store data it will replicated in 3, means it will store in different files in different machine to secure data.

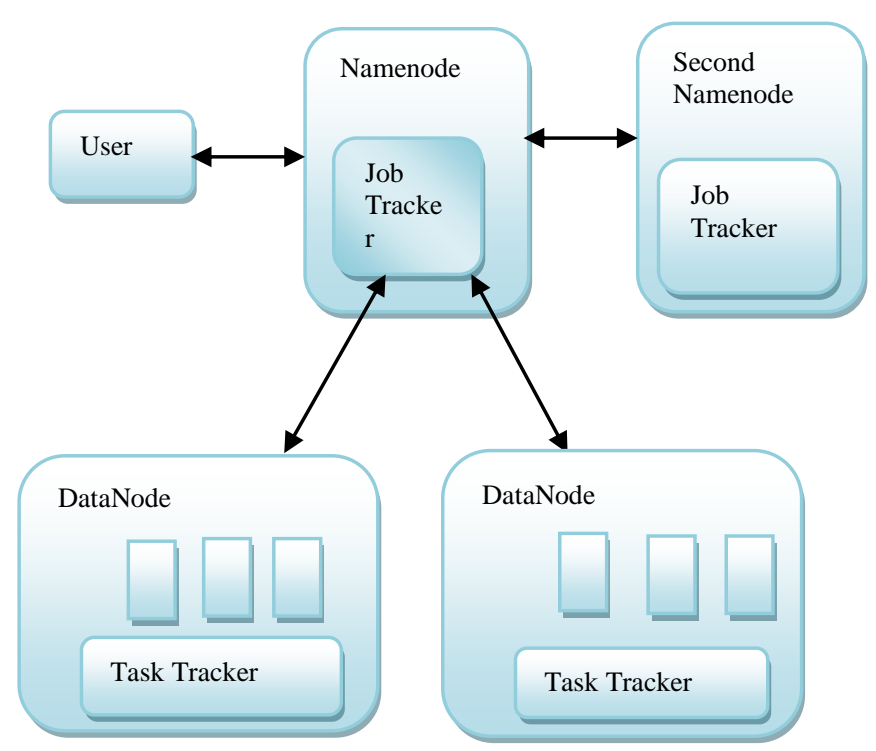

Figure 2: Big Data Storage Architecrure

In centralized environment there is only one master machine that holds all data means centralized storage. If centralized storage fails then whole system fails. But in Hadoop environment the storage done in distributed way. There is only metadata that hold whole information about the data which is stored on different location. Here metadata called NameNode.

The name node backup will be taken Secondary Namenode after some interval of time it will store to SSN. Here once we want to store data it will replicated in 3, means it will store in different files in different machine to secure data.

In centralized environment there is only one master node and multiple slave nodes. The master node holds all data. Any slave machine has storage device. All are connected to the master node to store and retrieve data. Here the storage has a limit if it go in large size like TB, PB, EB. The computing 
time will be huge. To overcome this problem hadoop system introduced.

In hadoop environment one master node and multiple slave nodes present. Here master node contains the Name Node (NN), Job Tracker (JT). Every node contains two part storage part and computation part. The NN is the metadata it hold the address of whole slave node where the actual data is stored. The JT is computation part here it will take the data from NN means address. And compute using task tracker. The slave node contains Task Tracker (TT) and Data Node (DN). The DD contains actual data or physical data. The TT contains computation on actual data. Here the storage done in different data node. The data is replicated to different slave nodes. So data loss problem will be solved. Any data loss or slave node crash other will be served. There is chance of master node loss. It is solved by Secondary Name Node (SNN). Every time interval the name node value will be copied into SSN so it will be secure.On each slave machine data node is there. It takes job from master and takes care of it. JobTracker will take of all process of master. JobTracker assigns jobs to TaskTracker in each slave machine using MapReduce Programming model. Processing of the data in each slave machine is handled by TaskTracker.

Now let us explain how data can be stored and processed by hadoop. Suppose we want use Hadoop to store 192MB of data adding a replication factor of 2 . Generally the replication factor is more then 3 .

In Hadoop platform before processing the data, file will be broken down into a size of $64 \mathrm{MB}$ or $128 \mathrm{MB}$ and that data blocks are moved to different slave nodes. Processing of the blocks will be done by running Hadoop framework. JobTracker will schedule the jobs of each node and TaskTracker processes the data. After completion of storage and processing of data the output is written back.

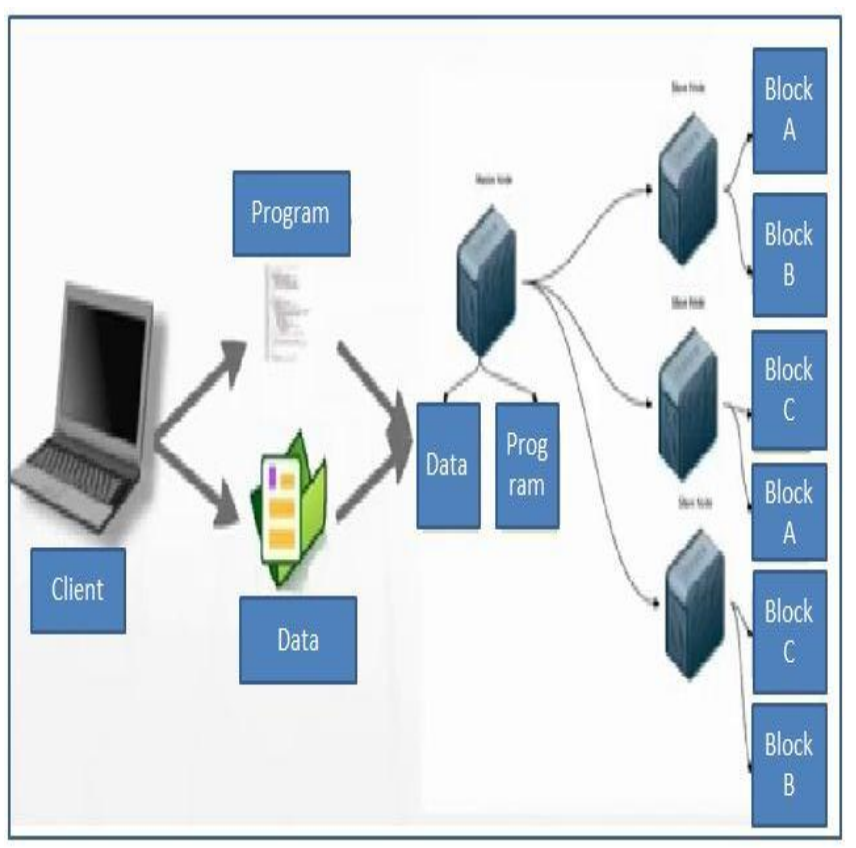

Figure 3: Storage and Process of Data in Cluster
$192 \mathrm{MB}$ of data will be divided into three blocks of size 64 MB (64MB *3=192MB). In this example we want replicate the data by the factor of 3 , to minimize the loss of data. In our example three slaves are available; Hadoop will replicate the block on different slave machines mainly to recover from dataloss by node failure. If one of the node fails we can recover from another machine.

Fig describes the following things,

1. Block $\mathrm{A} \rightarrow$ stored at slave 1 and 2

2. Block $\mathrm{B} \rightarrow$ stored at slave 1 and 3

3. Block $\mathrm{C} \rightarrow$ stored at slave 2 and 3

The storing, managing, analysis and processing will be performed by Hadoop itself and client will simply provide the data to the master node and replication factor.

\section{A. Related Technologies}

Here we are studying the different technologies used by Hadoop in Big Data storage system. It includes various components for Hadoop environment.

\section{B. Hadoop}

It is free available software and open source platform. It runs on "linux environment". Large quantity of data can be stored and processed. For example, if we want to store the whole organizations data into HADOOP, then software will devid ethe data into pieces and then spread across the different servers. Hadoop keeps track of the locations of all servers where data is stored. Apache Hadoop consists of many components like "Hadoop kernel, MapReduce, HDFS, Apache HIVE, HBase, Zookeep”.

\section{Hadoop Distributed File System (HDFS)}

It is a "fault tolerant storage system". It will store vast amount of data, scale up and also recover from loss of data. It creates a group of machines called as cluster and distributes work among the machines. If one of the machines fails, still it will operate without losing any data.it will divide the data into pieces called as "blocks" and stores it across the number of servers. It is having a default replication factor of 3 .

It is generally "expensive to build bigger servers" for handling large scale processing, but we can form a cluster by connecting many machines to a master machine.

Main tasks of Hadoop are:

1. Data is divided into pieces of uniform size 64 or 128 MB.

2. Those pieces forms a blocks and are distributed across cluster.

3. HDFS performs processing of the files.

4. Hadoop has inbuilt feature of replications and it will replicates to recover from hardware failure. 


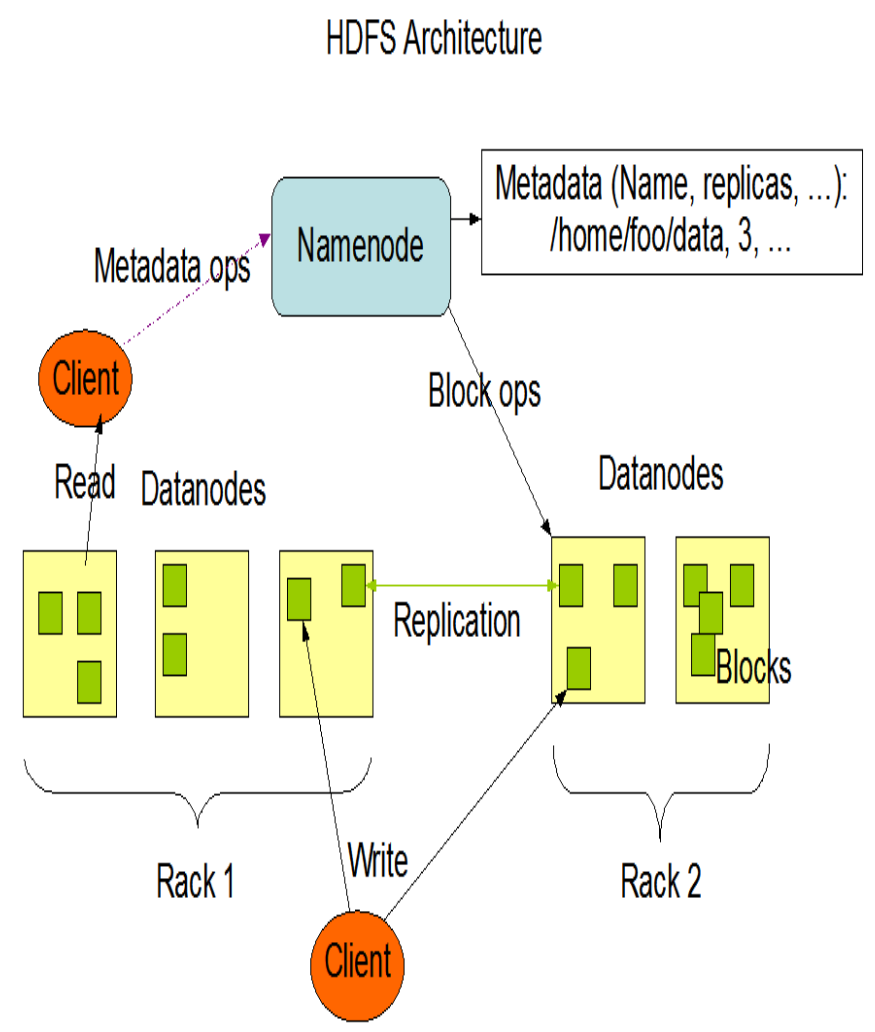

Figure 4: HDFS Architecture

HDFS architecture has a master angd slave format. It consists of a single NameNode and a number of DataNodes. NameNode acts as a master and distribute the tasks to DataNodes into fixed blocks of 64MB or $128 \mathrm{MB}$ size. There will be only one DataNode per cluster and it manages the storage. It executes operations like "opening, closing, and renaming". DataNode will perform read and write operations. DFS follows the master-slave architecture and it has the following elements. It manages system namespace. NameNode will perform the task provided by DataNode.

\section{Goals of HDFS}

1. Fault detection and recovery: It includes commodity hardware and failure of hardware component occurs repeatedly. It includes a mechanism for fault detection and recovery.

2. Large amount of data: It includes a large number of nodes to store vast amount of data.

\section{RESULTS AND DISCUSSION}

In the proposed model storage of data is important and it will show how data is stored in Cluster. The objective of the model is to reduce the loss of data and recovery of data using replication. Implementing the model with two or more slaves will reduce the loss of data by replicating the data into number of slaves. As the number of slaves increases loss of data will reduce. If there is only one slave machine then data will be replicated in one slave and loss of data is maximum.

\section{Cluster Summary}

7 files and directories, 1 blocks = 8 total. Heap Size is 31.57 MB / 966.69 MB (3\%)

$\begin{array}{llr}\text { Configured Capacity } & : & 35.18 \text { GB } \\ \text { DFS Used } & : & 28.01 \text { KB } \\ \text { Non DFS Used } & : & 7.4 \text { GB } \\ \text { DFS Remaining } & : & 27.78 \text { GB } \\ \text { DFS Used\% } & : & 0 \% \\ \text { DFS Remaining\% } & : & 78.96 \% \\ \text { Live Nodes } & : & 1 \\ \text { Dead Nodes } & : & 1 \\ \text { Decommissioning Nodes } & : & 0 \\ \text { Number of Under-Replicated Blocks } & : & 2\end{array}$

Fig

Figure 5: HDFS Cluster Summary

It will show the HDFS cluster summary means the overall information about the cluster. It shows the number of live nodes, dead nodes, amount of data stored, amount of data replicated

etc.

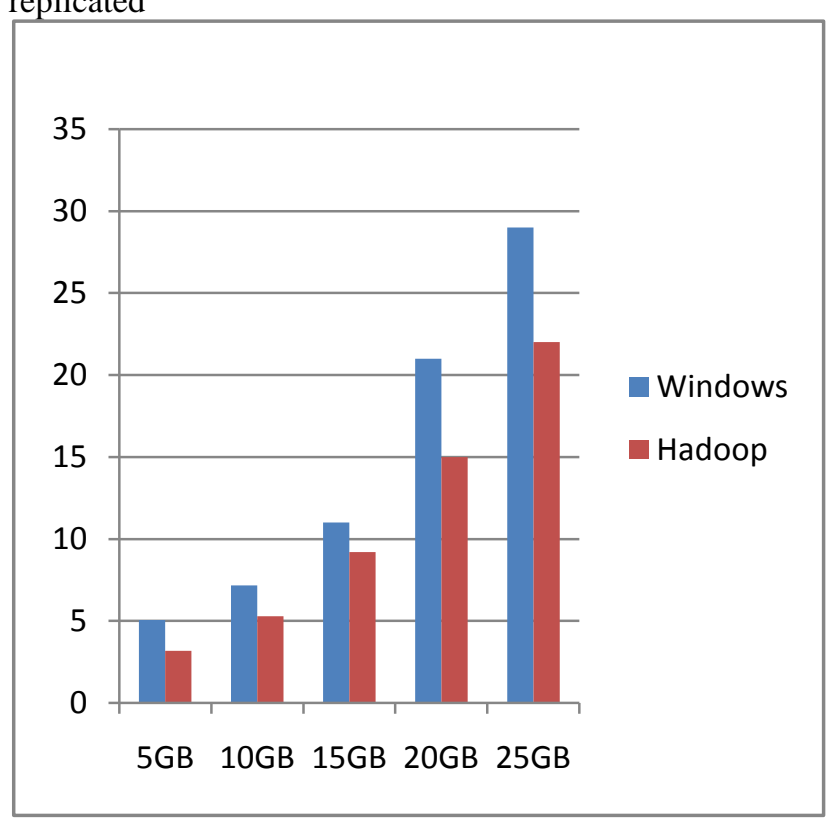

Figure 6: Performanace Chart

The performance of the system is observed. Here performance of the system is compared depending on the time taken to copy the data into the Hadoop and in normal platform. Hadoop can store data is less time comapare to other platfoems.

\section{CONCLUSION}

The important factor to store and retrieve data is system should be reliable, secure and fast computing. The mechanism presented in this thesis implements storage in efficient manner as the previous system fails to store unstructured and semi structured. Here the -storage of data is done in replication, so loss of data taken care here. If data stored in one slave fails 
then other slave will take care of that. The name node will take care of all data. It holds the meta data. All replicas will be stored in meta data. In case of processing it can process any type of data like structured semi structured and unstructured. In HDFS storing of data is very easier compared to the existing system.

Nowadays it is used in limited area like private network VPN. It can be extended to the cloud. There cloud plays storage systems. Cloud service provider become framework there we can store metadata. Data storage and processing get easier in cloud.

\section{REFERENCES}

[1] T. White, "Hadoop: The Definitive Guide", O'Reilly Media, Edition, 2012

[2] Intel It Center, "Planning Guide- Getting started with Big Data".

[3] Academia.edu, "Processing Big Data using Hadoop Framework".

[4] Robert D. Schneider," Hadoop for Dummies".

[5] Michael G. Noll. Running Hadoop on Ubuntu Linux (Single Node Cluster)

[Online]. Available: Website links: Apache Hadoop

[6] J. Dean and S. Ghemawat, "MapReduce: Simplified data processing on large clusters," in USENIXSymposium on Operating Systems Design and Implementation, San Francisco, CA, Pp. 137-150, 2004.

[7] A. Katal, M. Wazid and R.H. Goudar, "Big data: Issues, challenges, tools and Good practices", Noida, Pp. 404 - 409, 2013.

[8] Michael Di Stefano, "Distributed Data Management for Grid Computing", 2005.

[9] Y. Deng, F. Wang, N. Helian, S. Wu and C. Liao, "Dynamic and scalable storage management architecture for Grid Oriented Storage device", Parallel Computing, Vol. 34, Pp. 17-31, 2008.

[10] J. Lin, "MapReduce Is Good Enough?", The control project. IEEE Computer, Vol. 32, 2013. 